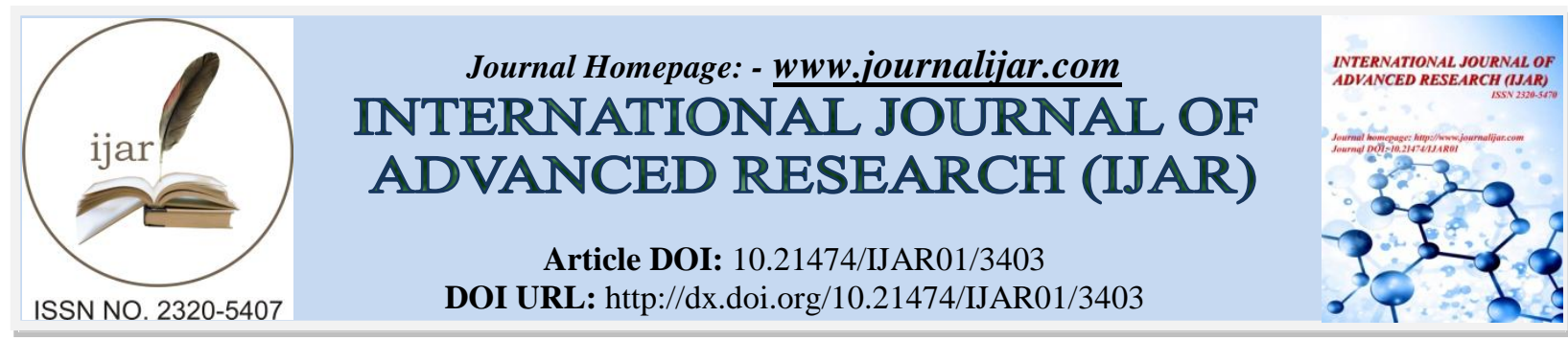

RESEARCH ARTICLE

\title{
PATHOLOGY BEYOND SLIDES-WHERE DO YOU THINK THE FUTURE LIES?
}

"Dr. Sarvesh Vijay ${ }^{1}$, Dr. Nanda Prasad ${ }^{2}$ and Dr. Yashwanth Reddy ${ }^{3}$.

1. PG-Student, Department Of Oral \& Maxillofacial Pathology, Sri Rajiv Gandhi College of Dental Sciences \& Hospital, Cholanagar, Hebbal, Bangalore, Karnataka, India.

2. Professor, Department Of Oral \& Maxillofacial Pathology, Sri Rajiv Gandhi College of Dental Sciences \& Hospital, Cholanagar, Hebbal, Bangalore, Karnataka, India.

3. Senior Lecturer, Department Of Oral \& Maxillofacial Pathology, Sri Rajiv Gandhi College of Dental Sciences \& Hospital, Cholanagar, Hebbal, Bangalore, Karnataka, India.

\section{Manuscript Info}

Manuscript History

Received: 10 December 2016

Final Accepted: 13 January 2017

Published: February 2017

Key words:-

Oral and Maxillofacial Pathology,

DigitalPathology,Diagnosis,Histopatholo gy,ImageAnalysis,Nanosensors,

Pharmacogenomics.

\section{Abstract}

Oral and Maxillofacial Pathology today is viewed as a speciality of dental sciences, which addresses the diagnosis and treatment of the disease of the oral and peri-oral regions. As medical practice, it covers all aspects of diagnostic methods on solid or fluid tissues based on laboratory techniques.It also covers the methodologies to transmit diagnostic data in an interpretable way to other physicians directly involved with patient treatment.It also manages tissue banks for research or diagnostic purposes. Cellular therapies from tissue banks or blood banks are increasingly becoming part of Oral and Maxillofacial Pathology.The object of investigation in histopathology is the digital slide, which is accessible throughout the world with no time or geographical limits.It permits the digital modelling of routine histological and/or cytological slide and it also allows measurements by using image analysis or stereology software packages. The electronic slide can be viewed, examined and diagnosed on a computer connected to a microscope, a new interface in diagnostic histopathology.This article describes the possible uses of digital slides, future of Oral and Maxillofacial Pathology beyond slides which by improving communication could have a positive effect on the entire health care system that will redefine how pathology will be practiced and the role of the Oral and Maxillofacial Pathologist in India in the near future.

Copy Right, IJAR, 2017,. All rights reserved.

\section{Introduction:-}

The four main aspects of applied medical information technology, which change the traditional systems of the entire health service are signal and data processing,digital modelling and interface optimisation. The information technology serving individual clinical specialties including Oral and Maxillofacial Pathology is changing at each of the four levels resulting in transformation of the communication paradigms. ${ }^{[1]}$ Digital pathology systems offer Oral and Maxillofacial Pathologists an alternate, emerging mechanism to manage and interpret information. They offer increasingly fast and scalable hardware platforms for slide scanning and software that facilitates remote viewing, slide conferencing, archiving, and image analysis. Digital modelling of routine histological and/or cytological slide is increasingly being implemented for direct patient care. ${ }^{[2]}$ Improvements in image quality, scan times, and image 
viewing browsers will hopefully allow Oral and Maxillofacial Pathologists to more seamlessly convert to digital pathology, much like our Oral Medicine and Radiology colleagues. ${ }^{[3]}$ Digital imaging in pathology has undergone an exponential period of growth and expansion catalyzed by changes in imaging hardware and gains in computational processing. Today, digitization of entire glass slides at near the optical resolution limits of light can occur in 60 seconds. ${ }^{[4]}$ Conventional histopathology is rapidly shifting towards digital integration. Will microscopes (and Oral and Maxillofacial Pathologists) soon be obsolete? Or are we dealing with just another image modality that leaves the core of tissue diagnosis intact ? ${ }^{[5]}$ This article provides an overview of current digital pathology beyond slides.

Future Of Oral and Maxillofacial Pathology Beyond Slides:-

This article explores trends within society and medicine which could significantly change Oral and Maxillofacial Pathology practice in India in near future the most important ones being advances in medical knowledge and advances in human technology.

\section{Advances in Medical Knowledge:-}

Disease is being understood increasingly in terms of molecular and sub- cellular processes which will have at least three major implications. The first and most promising will be the improvement to patient care as we can expect earlier diagnosis, more accurate prognosis and improved therapies as we better understand the disorders. ${ }^{[1-3]}$ The second implication will be increasing specialisation of medicine.A third consequence will be the drive towards personalised medicine which could result in shift from routine slide workload to a more potential shift. ${ }^{[4]}$

\section{Advances in Human Technology:-}

The next trend to consider is the rapid development of technology. Diagnostic tests are being refined which reveal the genomic and proteomic features of disease.Emergent new technologies impacting Oral and Maxillofacial Pathology are-Molecular Diagnostics at the histology level, Digital Diagnostics ,Bedside diagnostics, Nanosensors, Pharmacogenomics. ${ }^{[5]}$

Of these Digital Pathology will be most closely related for getting the most from the static image in the future.The current paradigm includes-Simple staining of histopathology sections (typically hematoxylin- eosin), IHC is used to identify expressed proteins by specific antibodies (one antibody per section). ${ }^{[6]}$ The problems arise when multiple markers/sections are needed, the amount of tissue available may not be enough for all markers. The future new paradigm may include application of multiple antibodies on the same section, using Quantum Dot photoemitters attached to each antibody species. ${ }^{[7]}$

Virtual Microscopy-Going beyond the microscope are current technologies under rapid development which aim to create a digitized computer file from complete scanning of a stained tissue section. ${ }^{[8]}$ The digitized file will be amenable to examination at any spot on a computer screen at different magnifications. This may replace the microscope as the basis for morphologic analysis of tissues. ${ }^{[3]}$

Histology based proteomics will provide a complete proteomics analysis connected to the histology of the tissue. This may form the practical applications for pathology practice as when in the recent future all proteins are mapped on standard mass, this approach will allow complete molecular determination of all proteins present in a section. ${ }^{[9]}$

Concurrent mapping a marker for endothelial cells and marker for a tumor marked together in different pseudocolors can determine vascular invasion of many oral lesions. Salivaomics technologies may contribute significantly in the identification of alterations in gene expression, transcription, protein coding and small molecules concentration, in biologic systems and may represent a novel and holistic approach in oral disease management including diagnosis, prognosis and monitoring. ${ }^{[3-5]}$ Elevated levels of circulating immune complexes (CIC) in the sera of oral cancer patients suggest that it contributes in evaluating the degree of malignancy, and provide an approach for a more precise test to predict prognosis of the disease which are far beyond slides only. ${ }^{[10]}$

\section{Advantages And Disadvantages Of Pathology Beyond Slides ${ }^{[2]}$}

Advantages-

- Facilitates rapid diagnosis.

- Cost effective.

- Helps leverage existing resources.

- Provides coverage for remote sites. 
- Useful for remote frozen sections.

- Useful for immediate fine- needle aspiration evaluation.

- Potential to improve patient care.

- Secondary consultation.

- Ability to retain original material at the host institution.

- Decreased turnaround time to retrieve, deliver, and file glass slides.

- Less effort required by support staff to handle consults.

- More rapid than courier services.

- No risk of slide loss or damage.

- Portability of the telepathologist.

- Real-time consultation.

- Possibility of simultaneous collaboration (teleconferencing).

- More timely response for patients and family awaiting a secondary review.

- Eliminates the need for multiple recuts; 1 slide may be scanned and viewed by an entire class.

- Avoids the risk of broken, damaged, lost, or unsatisfactory slides.

- Eliminates the need and cost for microscopes.

- Access to a wider variety of cases.

- Teleconferencing to multiple sites.

- Portability.

\section{Disadvantages-}

- May overextend existing resources (i.e consulting / covering pathologist).

- Difficult to handle multiple time sensitive requests arriving at the same time.

- Some cases may still require the pathologist to review the glass slide.

- May take longer than glass slide review.

- Potential for downtime.

- System maintenance required.

- Review of multiple slides including special stains for difficult cases may be difficult to review.

- Billing may be difficult, especially with missing information.

- Technical failures are possible.

- Poor image resolution compared with glass slides.

- Initial setup costs.

- Does not precisely simulate real-life evaluation of diagnostic material.

- Review of multiple slides or special stains may be difficult.

- Access to computers, higher network bandwidth, and/or monitors required in teaching environments.

\section{Conclusion:-}

To conclude we may state that Oral and Maxillofacial Pathology may not be limited to slides only in the near future as the amount of information that can be extracted from minute tissue and fluid samples using complex, automated and miniaturized devices will continue to increase. Sophisticated computer-based algorithms will provide assistance in integration of all information. Lab and tissue- based diagnostics will be increasing their capability to provide a safe guide to therapy. Enhanced imaging capabilities will allow groups of pathologists to share information on tissue based diagnostics. Pathology practitioners, blending knowledge of histopathology, disease related molecular processes and lab diagnostics, will be the integrators of information related to the molecular, biochemical and cellular processes underlying the patient's disease, complications and symptoms which may be far beyond slides. Will anybody ever, be able to do without us? The answer to this will always remain NO. 


\section{References:-}

1. Gombas P, Skepper JN, Krenacs T, Molnar B, Hegyi L. Past, present and future of digital pathology. Orv Hetil. 2004 Feb 22;145(8):433-43.

2. Cornish TC, Swapp RE, Kaplan KJ. Whole-slide Imaging: Routine Pathologic Diagnosis. Adv Anat Pathol. May $2012 ; 19(3): 152-59$.

3. Grimes GJ , McClellan SA, Goldman J, Vaughn GL, Conner DA, Kujawski E, McDonald J, Winokur T, Fleming W. Applications of virtual reality technology in pathology. Stud Health Technol Inform. 1997;39:31927.

4. Ghaznavi F, Evans A, Madabhushi A, Feldman M. Digital Imaging in Pathology: Whole-Slide Imaging and Beyond.2013; 8(1):5-12.

5. Brachtel E, Yagi Y. Digital imaging in pathology current applications and challenges. J Biophotonics. 2012 Apr ;5(4):327-35.

6. Al-Janabi S, Huisman A, Van Diest PJ. Digital pathology: current status and future perspectives. Histopathology. $2012 \mathrm{Jul}$; 61(1):19.

7. Jara Lazaro AR, Thamboo TP, Teh M, Tan PH. Digital pathology: exploring its applications in diagnostic surgical pathology practice. Pathology. 2010;42(6):512-8.

8. Rocha R, Vassallo J, Soares F, Miller K, Gobbi H. Digital slides: present status of a tool for consultation, teaching, and quality control in pathology. Pathol Res Pract. 2009;205(11):735-41.

9. Furness PN. The use of digital images in pathology. J Pathol. 1997 Nov;183(3):253-63.

10. Hamilton PW, Wang Y, McCullough SJ. Virtual microscopy and digital pathology in training and education. APMIS. 2012 Apr;120(4):305-15. 